\title{
Die Auswirkungen der COVID-19-Pandemie auf die psychische Gesundheit von Kindern, Jugendlichen, Familien und Paaren
}

Marie-Christin Hinteregger

Ein Zwischenresümee im Sommer 2021: Seit mehr als einem Jahr steht unser alltägliches Leben unter dem massiven Einfluss der COVID-19-Pandemie. Social Distancing, Ausgangsbeschränkungen, Testpflicht, FFP2-Masken, Home Office und Home Schooling - um nur ein paar der Begriffe zu nennen, die das vergangene Jahr geprägt haben - gehören längst gewissermaßen zu unserem Alltag und sind für die meisten von uns lange nicht mehr so befremdlich wie noch zu Beginn der Pandemie. Vielerorts lag das Augenmerk der Öffentlichkeit im letzten Jahr auf den gesundheitlichen Auswirkungen einer Infektion mit SARS-CoV2 sowie deren Vermeidung, in jüngster Vergangenheit finden jedoch auch die psychischen Auswirkungen der Pandemie sowie der Maßnahmen zu ihrer Eindämmung Beachtung in Forschung und Medien. Was im vergangenen Jahr unseren privaten wie auch beruflichen Alltag prägt, geht nicht spurlos an uns vorüber: Wir fürchten, uns mit dem Virus zu infizieren oder andere Menschen durch eine Ansteckung zu gefährden; die Pandemie konfrontiert uns unausweichlich mit unserer eigenen Vulnerabilität und Sterblichkeit. Wir empfinden Angst vor Kontrollverlust und existenzielle Ängste, die bei finanziellen Sorgen und der Sorge um unsere eigene sowie die gesamtwirtschaftliche Lage beginnen, und sich über Ängste die ausreichende (intensiv-)medizinische Versorgung betreffend bis hin zu Ängsten der Versorgungsknappheit erstrecken. Diese waren insbesondere im ersten Lockdown des vergangenen Jahres im Hinblick auf Lebensmittel und Gebrauchsgüter des täglichen Bedarfs wie Toilettenpapier und Seife deutlich spürbar.

Angst ist in dieser herausfordernden Zeit zu unserer ständigen Begleiterin geworden - doch was bedeutet das für uns? Als emotionaler Zustand steht Angst bzw. Furcht in Verbindung sowohl mit psychischen als auch mit physiologischen Reaktionen. Neben einem Gefühl der Anspannung, der Besorgtheit bzw. subjektiven Bedrohung, neben innerer Unruhe und Nervosität kommt es auch zu einer erhöhten Aktivität des autonomen Nervensystems sowie zu einem Anstieg von Blutdruck und Herzfrequenz 
(vgl. Dorsch Lexikon der Psychologie 2019, 2021). Angst stellt eine überlebensnotwendige Stressreaktion auf eine gefährliche Situation dar, indem das Gehirn Botenstoffe produziert, die die Abgabe von Hormonen der Nebennierenrinde anregen und in potenziell lebensbedrohlichen Gefahrensituationen zu einer Mobilisation der letzten Kraftreserven führen, um eine Kampf- oder Fluchtreaktion zu ermöglichen. Handelt es sich jedoch - wie im Fall der COVID-19-Pandemie - nicht um eine einmalige bzw. kurzfristige Gefahrensituation, sondern um eine länger andauernde oder gar stabile Veränderung unserer äußeren Lebenswelt, kann aus einer evolutionsbiologisch sinnvollen Notfallreaktion Dauerstress werden, der wiederum - erleben wir die Situation als nicht bewältigbar - stressbedingte psychische sowie körperliche Erkrankungen zur Folge haben kann (vgl. Hüther 2012). Hinzu kommt, dass durch die Maßnahmen zur Eindämmung der Pandemie viele jener Ressourcen und Möglichkeiten, die uns Einzelnen sowie auch ganzen Familiensystemen Entlastung bringen - so genannte Qualitätszeit, also unbeschwerte Freizeitaktivitäten, Urlaubsreisen, soziale Kontakte und vieles mehr - im vergangenen Jahr nur stark eingeschränkt bis gar nicht verfügbar waren. Die Zeiten, in denen ein dauerhaft angespanntes System also Erholung erfährt und Menschen die nötige Kraft für die Bewältigung ihrer alltäglichen Herausforderungen tanken können, mussten häufig und über lange Strecken hinweg zur Gänze ausfallen.

Auf die Auswirkungen, die diese drastischen Veränderungen unseres Alltags insbesondere auf Kinder, Jugendliche, Familien und Paare haben, möchte ich im vorliegenden Beitrag eingehen. Ich beziehe mich dabei insbesondere auf meine persönlichen Erfahrungen aus meiner klinisch-psychologischen Tätigkeit. Zusammen mit meinem Kollegen führe ich eine psychologisch-psychotherapeutische Praxis, in der wir uns auf die Therapie und Beratung von Kindern, Jugendlichen, Familien und Paaren spezialisiert haben; im Zuge unserer Tätigkeit haben wir in den vergangenen Monaten weitreichende Einblicke in die lebensweltlichen Veränderungen sowie die daraus resultierenden Veränderungen der psychischen Gesundheit und Lebenszufriedenheit unserer Klientinnen und Klienten erhalten. Im Folgenden möchte ich einige unserer Beobachtungen teilen, sie in Beziehung zur aktuellen Forschung setzen sowie auch einen Ausblick auf die Möglichkeiten des Umgangs mit dem sich deutlich zeigenden Anstieg psychischer Erkrankungen im Zusammenhang mit der COVID-19-Pandemie und den Maßnahmen zu ihrer Eindämmung geben. 


\section{Auswirkungen der COVID-19-Pandemie auf die psychische Gesundheit von Kindern und Jugendlichen}

Eine Studie der Donau-Universität Krems, durchgeführt in Kooperation mit der Medizinischen Universität Wien und dem Bundesministerium für Bildung, Wissenschaft und Forschung, präsentiert uns ein Ergebnis, das als höchst alarmierend angesehen werden kann: Die psychische Gesundheit von Schülerinnen und Schülern zwischen 14 und 20 Jahren hat sich drastisch verschlechtert. 64 Prozent von ihnen leiden unter klinisch relevanten Symptomen einer Essstörung, mehr als die Hälfte zeigt depressive Symptome und 50 Prozent der Jugendlichen leiden unter Ängsten. Auch die Auftretenshäufigkeit von Schlafstörungen hat zugenommen, bereits ein Viertel aller Schülerinnen und Schüler ist davon betroffen. Besonders deutlich zeigen sich die Auswirkungen psychischer Belastung im Bereich der Suizidalität: 16 Prozent der Jugendlichen haben täglich oder an mehr als der Hälfte der Tage suizidale Gedanken. Die Ergebnisse sind äußerst besorgniserregend, denn die Häufigkeit von depressiven Symptomen und Angstsymptomen hat sich damit im Vergleich zum Zeitraum vor der COVID-19-Pandemie bereits verfünf- bis verzehnfacht (vgl. Pieh et al. 2021).

Diese Studienergebnisse decken sich traurigerweise mit dem, was wir im Alltag unserer klinisch-psychologischen und psychotherapeutischen Praxis beobachten: Kinder und Jugendliche reagieren auf die Pandemie sowie auf die Maßnahmen, die zu ihrer Eindämmung gesetzt wurden - und vielen von ihnen scheinen die gesunden, nicht-destruktiven bzw. nichtselbstschädigenden Reaktionsmöglichkeiten schlichtweg ausgegangen zu sein. Im vergangenen Jahr waren es insbesondere zwei Copingstrategien, die uns als Antwort auf Home Schooling und Social Distancing in unserer Praxis vorrangig begegneten und die sich in diesem Zusammenhang am besten in internalisierende und externalisierende Verhaltensmuster unterteilen lassen. Dabei ist jedoch vorauszuschicken, dass es sich in beiden Fällen um unvollständige Copingstrategien handelt, also um Bewältigungsversuche, die zwar als gesunde (im Sinne von: verständliche) Reaktion auf eine ungesunde Situation gelten können, diese jedoch nicht zu verändern vermögen und so letztlich wiederum selbst in einer ungesunden Situation für die Betroffenen resultieren.

\subsection{Internalisierende Verhaltensmuster}

Sprechen wir von internalisierenden Verhaltensmustern oder internalisierendem Problemverhalten, so meinen wir Verhaltensauffälligkeiten, die 
primär nach innen gerichtet sind. Der Begriff der internalisierenden Störung stellt somit einen Sammelbegriff für Depressionen und Angststörungen im Kindes- und Jugendalter dar. Da internalisierende Verhaltensmuster durch überkontrollierte Verhaltensstrategien gekennzeichnet sind und sich durch passiv-defensives und vermeidendes Verhalten sowie Zurückhaltung im Sozialkontakt zeigen, gehen sie oftmals auch mit psychosomatischen Störungen, Störungen des Essverhaltens sowie Zwangsstörungen einher (vgl. Pschyrembel online 2016).

Internalisierende Verhaltensmuster beobachten wir im vergangenen Jahr verstärkt bei Kindern und Jugendlichen im Alter zwischen zehn und 18 Jahren. Dabei sind depressive und Angstsymptome vorherrschend, diese werden oftmals von selbstverletzendem Verhalten und suizidalen Gedanken begleitet. Besonders die Auftretenshäufigkeit selbstdestruktiver Verhaltensweisen und suizidaler Gedanken hat im Vergleich zum Zeitraum vor Beginn der Pandemie stark zugenommen. Auffallend ist dabei, dass die auftretenden Symptome bei den meisten von uns behandelten Kindern und Jugendlichen in kausalem Zusammenhang zur Schulsituation im vergangenen Jahr stehen: Die Schülerinnen und Schüler äußern Überforderung mit den Lehrinhalten sowie deren Umfang, geringere wahrgenommene Unterstützung durch die Lehrkräfte im Home Schooling, fehlende Motivation durch mangelnden Einbezug beim Online-Unterricht sowie fehlende soziale Kontakte mit Mitschülerinnen und Mitschülern. Bei vielen von ihnen haben sich die Noten im vergangenen Jahr im Vergleich zum Zeitraum vor der Pandemie verschlechtert, Leistungsbereitschaft und Motivation haben deutlich abgenommen. Eine besondere Herausforderung stellte für viele Schülerinnen und Schüler auch der Wechsel von der Primar- in die Sekundarstufe im Herbst des vergangenen Jahres dar: Sie hatten aufgrund der geringen Präsenzzeit zu Beginn des Wintersemesters keine Gelegenheit, den Schulbetrieb, die Lehrkräfte oder die Mitschülerinnen und Mitschüler persönlich kennenzulernen, was eine gelungene Umstellung für viele von ihnen deutlich erschwerte.

Um sich der belastenden Gesamtsituation zu entziehen, fliehen derzeit viele Kinder und Jugendliche, die zu internalisierenden Verhaltensmustern tendieren, in fiktive Welten, was das Risiko einer Mediensucht erhöhen kann (vgl. Pieh et al. 2021, Hanke et al. 2021). Andere wiederum reagieren auf die unkontrollierbare Situation im Außen mit Zwangshandlungen, die zumindest im Innen eine Illusion der Kontrollierbarkeit erzeugen und von den Kindern und Jugendlichen meist als im Moment angstmindernd und spannungsreduzierend, zugleich aber langfristig als belastend und stigmatisierend erlebt werden. Eine ähnliche, von den Betroffenen in der Regel als akut spannungslösend empfundene, jedoch 
langfristig problematische Copingstrategie stellt auch autoaggressives bzw. selbstverletzendes Verhalten dar. In beiden Fällen - sowohl bei Zwangshandlungen als auch bei Selbstverletzung - handelt es sich um unvollständige Bewältigungsversuche, die den Betroffenen nur kurzzeitig subjektiv empfundene Erleichterung verschaffen. Ziel einer psychologischen Therapie bzw. Psychotherapie kann daher das Verfügbarmachen und Einüben von alternativen, bestenfalls gesunden bzw. weniger schädlichen Bewältigungsstrategien sein.

\subsection{Externalisierende Verhaltensmuster}

Expansive bzw. externalisierende Verhaltensstörungen (vgl. Pschyrembel online 2016) zeigen sich als nach außen gerichtete Verhaltensauffälligkeiten im Kindes- und Jugendalter wie beispielsweise Störungen des Sozialverhaltens, Störungen mit oppositionellem Trotzverhalten oder die Aufmerksamkeitsdefizithyperaktivitätsstörung (ADHS). In unserer Praxis beobachten wir externalisierende Verhaltensmuster vorrangig bei Schulkindern im Alter bis zu zehn Jahren. Nun sei an dieser Stelle angemerkt, dass das gehäufte Vorkommen externalisierender Verhaltensmuster nicht auf eine Erhöhung der generellen Prävalenzrate von Verhaltensstörungen und ADHS im Kindes- und Jugendalter als Auswirkung der COVID-19-Pandemie zurückzuführen ist. Vielmehr sind die Auswirkungen der Pandemie und der ergriffenen Maßnahmen als eine Art Katalysator zu verstehen, der die bereits vorhandenen Symptomatiken verstärkt und in ihrer Genese beschleunigt. So leiden Kinder beispielsweise keineswegs aufgrund der Pandemie an ADHS; sie leiden jedoch aufgrund des Home Schoolings häufig unter dem Fehlen einer für die Betroffenen so wichtigen Tagesstruktur sowie aufgrund der einschränkenden Maßnahmen meist auch unter einem Bewegungsmangel, der im Kontext dieses Störungsbildes besonders drastische Auswirkungen zeigt und oftmals dazu führt, dass die Agitation nicht ausgelebt werden kann, sich stattdessen aufstaut und sich als unkontrollierte Aggression entlädt. Dies zeigt sich in hyperaktiven sowie aggressiven Verhaltensauffälligkeiten entweder in der Schule, zuhause oder in beiden Bereichen - und resultiert nicht zuletzt bei einigen der von uns behandelten Kinder darin, dass sie die verpflichtenden COVID-Tests im Unterricht verweigern.

Externalisierende Störungen haben im Gegensatz zu den internalisierenden, nach innen gerichteten Verhaltensmustern den Vorteil, dass sie vom Umfeld der betroffenen Kinder und Jugendlichen relativ leicht erkannt werden. Auch ist der Leidensdruck von Eltern, Lehrkräften und 
anderen Personen aus dem Bezugssystem hoch, da externalisierende Störungen einen deutlich spürbaren Einfluss auf das soziale Miteinander haben. Während internalisierende Störungen, die sich nach außen hin eher durch Traurigkeit und sozialen Rückzug bemerkbar machen, häufig übersehen werden, ist dies bei externalisierenden Verhaltensmustern selten der Fall; sie werden aufgrund ihrer Auffälligkeit eher wahrgenommen. Die betroffenen Kinder und Jugendlichen erhalten infolgedessen auch umso wahrscheinlicher Unterstützung in Form von psychologischer Therapie bzw. Psychotherapie. Diese besteht zu einem wesentlichen Teil aus der Beratung der Eltern im Hinblick auf Tagesstrukturierung, Sensibilisierung für die Bedürfnisse der Kinder, das Setzen von Grenzen und die Förderung gesunder Stressbewältigungsstrategien.

\subsection{Risikofaktor Home Schooling}

Was für manche Kinder und Jugendliche der Leistungsdruck ist, die guten Noten von früher halten zu wollen, es aber im Kontext des Home Schoolings nicht zu schaffen, ist für manch andere die reale Angst, eine Klasse wiederholen zu müssen. Spätestens an dieser Stelle kommen auch die Eltern mit ins Spiel, die im vergangenen Jahr nicht nur ihrer eigenen Rolle gerecht werden, sondern zum Teil auch in jene von Pädagoginnen und Pädagogen schlüpfen mussten. Oftmals selbst unter prekären Arbeitsbedingungen im Bereich der medizinischen Versorgung, der Pflege oder des Handels beschäftigt, von drohendem oder tatsächlichem Arbeitsplatzverlust betroffen oder im Home Office auf begrenztem Raum mit den anderen Familienmitgliedern, bemühen sich Eltern um den Spagat zwischen ihren Rollen als Motivator*innen, Kontrolleur*innen, Erzieher*innen, Seelentröster*innen und als interimistische Lehrkräfte. Dabei sind in den meisten Fällen sowohl ihre zeitlichen Ressourcen als auch ihre Möglichkeiten, bei komplexen Aufgabenstellungen in der Sekundarstufe unterstützen zu können, limitiert. Familien geraten in derartigen Überforderungssituationen nur allzu leicht in eine Negativspirale, die aus eigener Kraft nur schwer zu verlassen ist: Die Schülerinnen und Schüler sind lust- und antriebslos, überfordert und verzweifelt, die Eltern sind sowohl bemüht als auch gewissermaßen verpflichtet zu unterstützen. Es kommt zu Auseinandersetzungen in Bezug auf schulische Belange und zu negativen Beziehungserfahrungen, die sich häufen. Zugleich schränken die wiederkehrenden Maßnahmen im Zusammenhang mit der COVID-19-Pandemie die Möglichkeiten, unbeschwerte Zeit als Familie miteinander zu verbringen, teilweise drastisch ein - man denke nur an die Zeiten gesperrter 
Kinderspielplätze und strikter Ausgangsbeschränkungen bei Tag. Eltern geraten so unversehens in die Rolle, ihre kaum noch zu motivierenden Kinder regelrecht zum Unterricht zwingen zu müssen, um ihrer Verantwortung nachzukommen, was zu Widerstand bei den Kindern führt und sich häufig darin äußert, dass ihre depressiven und Angstsymptome bzw. aggressiven Verhaltensmuster unter Druck weiter zunehmen. Das Thema Schule wird so Tag für Tag zu einem Kampf zwischen Kindern und Eltern, der meist in Auseinandersetzungen und Streit resultiert und die familiären Spannungen sukzessive erhöht. Nach einem Jahr Pandemie beobachten wir, dass sich der Kontakt vieler Eltern mit ihren Kindern inzwischen beinahe ausschließlich auf schulische Belange beschränkt und sich dabei - kaum überraschend - für beide Seiten deutlich belastet und negativ behaftet darstellt. Die Beziehung ist problemzentriert und kreist um das belastende Thema; Erholungsphasen in Form von unbeschwerter Qualitätszeit, die in jeder Familie, noch mehr jedoch in von psychischen Erkrankungen betroffenen Bezugssystemen eine wesentliche Ressource darstellen, existieren nur eingeschränkt und entlang der jeweils aktuellen Maßnahmen. Eine wesentliche und meist initiale Intervention im Rahmen einer psychologischen Therapie bzw. Psychotherapie ist daher die bewusste (Wieder-)Einführung gemeinsamer Zeit von Eltern und Kindern bzw. Jugendlichen abseits der schulischen Verpflichtungen zur Entlastung der angespannten Familiensituation.

An dieser Stelle sei darauf hingewiesen, dass es sich bei den klinischpsychologischen und psychotherapeutischen Leistungen in unserer Praxis um Privatleistungen handelt. Dies legt nahe, dass die hier beschriebenen Beobachtungen in Bezug auf die drastische Verschlechterung der psychischen Gesundheit von Kindern und Jugendlichen während der COVID-19Pandemie sich zwar auf Familien mit unterschiedlichem sozioökonomischem Hintergrund beziehen, diese dennoch tendenziell keiner akut armutsgefährdeten Risikogruppe angehören. Haushalte mit Kindern stellen jedoch generell eine solche Risikogruppe dar; vor allem alleinerziehende Eltern und Mehrpersonenhaushalte mit mehr als drei Kindern weisen in Österreich ein erhöhtes Armutsrisiko von 25 Prozent auf (vgl. Stoppacher/Saurug 2018). Dies hat weitreichende Auswirkungen, die die psychische Gesundheit von Kindern und Jugendlichen zusätzlich negativ beeinflussen können, so beispielsweise fehlende finanzielle Mittel, um die notwendige technische Ausstattung für das Home Schooling zur Verfügung zu stellen, beengten Wohnraum und - besonders für Alleinerziehende die teilweise Unvereinbarkeit von existenziell notwendiger Berufstätigkeit und erforderlicher Kinderbetreuung in Zeiten des Home Schoolings. 


\section{Das Familiensystem als Klient in der psychologischen Therapie bzw.} Psychotherapie

Wie bereits aus den vorangegangenen Zahlen und Beobachtungen deutlich wird, sind Familien stark betroffen von den vielfältigen Auswirkungen, die die COVID-19-Pandemie auf die psychische Gesundheit hat. Dies bezieht sich auf Kinder und Jugendliche, aber ebenso auf Erwachsene: Mehrere Studien aus dem europäischen Raum, darunter auch eine der Donau-Universität-Krems, zeigen, dass die psychische Dauerbelastung im vergangenen Jahr zu drastischen Anstiegen unterschiedlicher Symptomatiken und einer deutlichen allgemeinen Verschlechterung der psychischen Gesundheit sowie der Lebenszufriedenheit geführt hat. Etwa ein Viertel der erwachsenen Bevölkerung leidet unter depressiven Symptomen, von Angstsymptomen sind 23 Prozent betroffen und 18 Prozent leiden unter Schlafstörungen. Bei jungen Erwachsenen im Alter zwischen 18 und 24 Jahren ist sogar bereits die Hälfte von depressiven Symptomen betroffen. Besonders alarmierend ist auch die starke Zunahme von Fällen schwerer Depression, die sich im vergangenen Jahr verzehnfacht haben (vgl. Dale et al. 2021).

Besonders stark betroffen von den psychischen Auswirkungen der COVID-19-Pandemie sind jene Personen, die bereits davor an einer psychischen Erkrankung litten: Die Wahrscheinlichkeit, erneut klinisch relevante Symptome zu entwickeln, ist während der Pandemie etwa doppelt so hoch (vgl. Schröder 2021). Auch wir konnten beobachten, dass psychische Vorerkrankungen wie Depressionen und Schlafstörungen, Störungen des Essverhaltens sowie Zwangsstörungen insbesondere in Zeiten der Ausgangsbeschränkungen erneut auftraten oder die Symptome sich häufiger bzw. intensiver zeigten. Für Menschen, die aufgrund ihrer Geschichte also bereits vorbelastet sind und eine höhere Vulnerabilität für das (erneute) Auftreten psychischer Erkrankungen aufweisen, erscheinen entsprechende Präventionsmaßnahmen sowie das Erlernen gesunder Bewältigungsstrategien daher umso wichtiger.

Anhand der Befunde wird deutlich, dass sich die Auswirkungen der Pandemie innerhalb von Familien tendenziell potenzieren, indem sowohl Eltern als auch Kinder von psychischen Belastungen betroffen sein können und sich die Belastung einzelner Familienmitglieder zugleich stets auch im gesamten Familiensystem niederschlägt. Auch spielt die Paarbeziehung der Eltern eine wesentliche Rolle im Hinblick auf die Familiengesundheit: Etwa die Hälfte aller Paare in Österreich lebt mit Kindern (vgl. Statistik Austria 2018) und sowohl für sie als auch für kinderlose Paare und Eltern erwachsener Kinder stellen die Erfordernisse des vergangenen Jahres 
häufig eine Zerreißprobe dar. Erneut zeigt sich in der klinisch-psychologischen bzw. psychotherapeutischen Praxis, dass die Pandemie sowie die Maßnahmen zu ihrer Eindämmung wie ein Katalysator wirken, der die Entstehung von Paarkonflikten beschleunigt und bereits vorhandene Konflikte schneller und deutlich heftiger eskalieren lässt. Viele Paare berichten von vermehrten Auseinandersetzungen und von fehlender Qualitätszeit - sowohl mit der Familie als auch zu zweit; sie vermissen unbeschwerte Auszeiten in Form von Ausflügen oder Urlaubsreisen. Während in Zeiten des Lockdowns kein Abendessen im Restaurant in ruhiger Zweisamkeit möglich war, finden die meisten Paare auch in Zeiten geöffneter Gastronomiebetriebe nur in seltenen Ausnahmefällen Personen aus dem familiären Umfeld, um auf die Kinder aufzupassen. Großeltern und andere Angehörige, so sie denn in der Nähe der Familie wohnen, kommen aufgrund ihrer Zugehörigkeit zu einer Risikogruppe meist nicht in Frage, was neben den fehlenden innerfamiliären Sozialkontakten auch in einer als mangelhaft wahrgenommenen Unterstützung bei vielen Eltern resultiert. Außerfamiliäre Kinderbetreuung lässt sich für die meisten Eltern in Zeiten der Pandemie ebenso schwer bis teilweise unmöglich organisieren, was zur Folge hat, dass viele von ihnen zum Wohl ihrer Kinder unter Aufbringung großer Anstrengung zwischen verschiedenen Rollen hin- und herwechseln, letztlich jedoch als Paar kaum noch Raum haben und, wie wir häufig in der Beratung feststellen, bereits resigniert haben, diesen auch für sich zu beanspruchen und einzufordern.

Zwar sind wir in unserer Praxis im Rahmen der Paar- und Familientherapie derzeit nicht verstärkt mit häuslicher Gewalt konfrontiert, dass es hier im vergangenen Jahr zu einer Zunahme gekommen ist, ist dennoch evident (vgl. Bundesministerium für Inneres/Bundeskriminalamt 2020). Was wir hingegen im Zusammenhang mit der Pandemie beobachten können und was von vielen Paaren thematisiert wird, ist eine deutliche Rückbesinnung auf tradierte Werte und „klassische“ Rollenbilder, die sich insbesondere in der Aufgabenverteilung im Hinblick auf Haushaltsführung und Kindererziehung zeigt. Es scheint, als mache die aktuelle Situation Menschen konservativer, denn selbst in Beziehungen bzw. Familien, in denen abseits der Pandemie generell Gleichberechtigung und Arbeitsteilung in jeglichen Bereichen herrschen, sind Frauen entsprechend traditionellen Geschlechterstereotypen nun wieder verstärkt für Haushalt und Kinder verantwortlich, während Männer die Rolle der erwerbstätigen Ernährer einnehmen. Diese Werteverschiebung, die derzeit in vielen Paarbeziehungen und Familien beobachtbar ist, kann als Reaktion auf die Ängste und Unsicherheiten gedeutet werden, die die aktuell herausfordernde Zeit mit sich bringt: Wir sehnen uns nach Stabilität, nach Sicherheit, nach 
Kontrolle und etwas Bekanntem, an dem wir uns festhalten können. Traditionelle Werte, die uns meist wohlvertraut sind, werden dafür intuitiv herangezogen - was letztlich auch eine kurzfristig zu Erfolg führende Copingstrategie darstellt und den massiven Stress widerspiegelt, den wir im Kontext der Pandemie zu bewältigen versuchen. Diese Beobachtungen decken sich mit den Ergebnissen einer amerikanischen Studie, die zeigt, dass die Pandemie und die mit ihr verbundenen Maßnahmen zu einer - wenn auch nur geringen - verstärkten Zustimmung zu traditionellen Geschlechterrollen führen (vgl. Rosenfeld/Tomiyama 2020). Auch eine Studie der Universität Salzburg in Kooperation mit der Universität Graz und der Universität Linz gelangt zu ähnlichen Erkenntnissen: Seit Beginn der COVID-19-Pandemie ist ein stetiger Aufwärtstrend im Hinblick auf konservative Werte erkennbar; Konformität hat als Grundwert in der Bevölkerung an Bedeutung gewonnen, wohingegen auf Unabhängigkeit und Hedonismus tendenziell eher verzichtet wird (vgl. Aschauer et al. 2020). Was auf den ersten Blick wie ein emanzipatorischer Rückschritt anmutet, spiegelt auch laut Rosenfeld und Tomiyama (2020) den Wunsch nach Stabilität in einer so unsicheren Zeit wider und soll keine ernsthafte Gefahr für die bisherigen Errungenschaften der Emanzipation, Gleichbehandlung und Gleichberechtigung bedeuten, sondern lediglich eine vorübergehende Reaktion auf die äußeren Umstände sein. Die generelle politische Gesinnung bleibt laut ihren Untersuchungen von diesen Umständen bis dato gänzlich unberührt, was zumindest die Hoffnung aufrecht hält, dass sich die derzeitigen Rollenbilder künftig wieder wandeln werden.

\section{Gesunde Bewältigungsstrategien im Umgang mit der COVID-19-Pandemie}

Eltern wie auch Lehrkräfte stellen in Anbetracht der erschreckenden Zahlen und Fakten die berechtigte Frage nach Maßnahmen, die zur Verbesserung der psychischen Gesundheit von Kindern und Jugendlichen beitragen. Hier sei aus klinisch-psychologischer Sicht in erster Linie die Sensibilisierung des gesamten Bezugssystems von Schülerinnen und Schülern genannt, die dazu beitragen soll, Bedürfnisse wahrzunehmen, Probleme rechtzeitig zu erkennen und entsprechende Unterstützungsangebote entweder selbst zu ermöglichen oder in einem professionellen Setting zu organisieren. Unterstützung beginnt in diesem Zusammenhang bereits beim offenen Gespräch über die Gefühle und Wahrnehmungen während der Pandemie und beinhaltet Achtsamkeit und Verständnis für die Ängste und Sorgen von Kindern und Jugendlichen. Die eigenen Gefühle wahrzunehmen, sie zu benennen und sie innerhalb der Familie und weiterer 
Bezugssysteme zu besprechen stellt einen wichtigen Faktor bei der Aufrechterhaltung bzw. Verbesserung psychischer Gesundheit dar. Eltern und Lehrkräfte sollten daher die psychischen Belastungen von Kindern und Jugendlichen - aber auch aller anderen Menschen in ihrem Umfeld ernst nehmen. Spätestens bei den genannten und häufig beobachteten internalisierenden und externalisierenden Verhaltensauffälligkeiten und Symptomen ist professionelle Unterstützung im Rahmen von psychologischer Therapie bzw. Psychotherapie dringend angeraten.

Eine weitere wichtige Rolle bei der Verbesserung der psychischen Gesundheit spielen - für Kinder und Jugendliche sowie auch gleichermaßen für Erwachsene - regelmäßige Sozialkontakte, ausreichend körperliche Bewegung an der frischen Luft und Möglichkeiten, aufgestaute Energie und Aggressionen in konstruktiver, nicht-schädigender Weise abbauen zu können. Was sich für Erwachsene am Ehesten unter den Begriffen der Achtsamkeit und Selbstfürsorge subsumieren lässt, ist insbesondere für Kinder und Jugendliche von der Achtsamkeit und Fürsorge ihres Bezugssystems abhängig. Im Rahmen dieses Beitrages muss von einer Vertiefung des Themas Aggression aus Platzgründen abgesehen werden, es sei an dieser Stelle jedoch gesagt: Bei Aggression handelt es sich um eine lebenswichtige Kraft, die uns Menschen innewohnt und die aufgrund fehlender gesellschaftlicher Akzeptanz kaum Platz in unserem modernen Leben findet. Dabei wird Aggression immer dann zum Problem, wenn keine konstruktiven Strategien zu ihrem Abbau verfügbar sind und sie sich stattdessen aufstaut, um sich später unkontrolliert und destruktiv in Zerstörung, Gewalt, autoaggressivem Verhalten u. Ä. zu entladen. Auch Aggression kann als eine gesunde Reaktion auf ungesunde Umgebungsbedingungen, wie sie die COVID-19-Pandemie und die damit in Zusammenhang stehenden Maßnahmen darstellen, verstanden werden. Ohne entsprechende Abbaumöglichkeiten sowie durch zunehmenden Stress in anderen Lebensbereichen kann es letztlich zu autoaggressivem Verhalten oder gewalttätigen Übergriffen an anderen Menschen sowie zur Zerstörung des eigenen oder fremden Eigentums kommen. Dem kann durch Sensibilität für die eigenen Emotionen, körperliche Bewegung und Sport, aber auch durch andere, individuell unterschiedlich gestaltete Maßnahmen der Selbstfürsorge entgegengewirkt werden.

Für Familien ist insbesondere ein Ausgleich zu spannungsgeladenen und konfliktbehafteten Themen im Zusammenhang mit Ausbildung und Schule sinnvoll und notwendig: gemeinsames Kochen und Backen, Gesellschaftsspiele, Ausflüge in die Natur - was immer Eltern und Kindern Freude bereitet und ihnen eine unbeschwerte Zeit miteinander ermöglicht, sollte neben den Verpflichtungen des Alltags Platz finden und bewusst 
eingeplant werden. Idealerweise können Entscheidungen dahingehend gemeinsam getroffen und Kompromisse geschlossen bzw. die Interessen und Wünsche aller in etwa ausgewogen berücksichtigt werden, um einen Ausgleich zu den belastenden Auswirkungen der Pandemie im Alltag zu schaffen.

Eine generell positive Einstellung zum Leben zeigt sich sowohl im Alltag als auch in krisenhaften Situationen als unterstützend für die psychische Gesundheit, weshalb sich - insbesondere in Zeiten der Pandemie eine bewusste Fokussierung auf individuelle sowie familiäre Stärken, Ressourcen und Möglichkeiten sowie auf gemeinsame positive Zukunftsbilder und bereits erfolgreich bewältige Herausforderungen lohnt.

\section{Literatur}

Aschauer, Wolfgang/Seymer, Alexander/Prandner, Dimitri/Baisch, Benjamin/Hadler, Markus/Höllinger, Franz/Bacher, Johann: Values in Crisis Austria (SUF edition) (2020), https://doi.org/10.11587/H0UJNT [abgerufen am 14.07.2021].

Bundesministerium für Inneres \& Bundeskriminalamt: Analyse zu häuslicher Gewalt während des Corona-Lockdowns (2020), https://bmi.gv.at/bmi_documents/ 2536.pdf [abgerufen am 24.05.2021].

Dale, Rachel/Budimir, Sanja/Probst, Thomas/Stippl, Peter/Pieh, Christoph: Mental Health during the COVID-19 Lockdown over the Christmas Period in Austria and the Effects of Sociodemographic and Lifestyle Factors, in: International Journal of Environmental Research and Public Health 18/7 (2021), https://doi.or g/10.3390/ijerph18073679 [abgerufen am 23.08.2021].

Dorsch Lexikon der Psychologie: Stichwort Furcht (2019), https://dorsch.hogrefe.c om/stichwort/furcht [abgerufen am 24.05.2021].

Dorsch Lexikon der Psychologie: Stichwort Angst (2021), https://dorsch.hogrefe.co $\mathrm{m} / \mathrm{stichwort/angst} \mathrm{[abgerufen} \mathrm{am} \mathrm{24.05.2021].}$

Hanke, Kai/Hofmann, Holger/Jonas, Cornelia/Kamp, Uwe/Krause, Torsten/Krüger, Thomas/Ohlmeier, Nina/Pohle, Sophie: Kinderreport Deutschland 2021, hrsg. vom Deutschen Kinderhilfswerk e. V. (2021), https://www.dkhw.de/fileadmin/R edaktion/1_Unsere_Arbeit/1_Schwerpunkte/2_Kinderrechte/2.29_Kinderreport _2021/Kinderreport_2021.pdf [abgerufen am 14.07.2021].

Hüther, Gerald: Biologie der Angst. Wie aus Streß Gefühle werden, Göttingen: Vandenhoeck \& Ruprecht 2012.

Pieh, Christoph/Plener, Paul/Probst, Thomas/Dale, Rachel/Humer, Elke: Health in Adolescents during COVID-19-Related Social Distancing and Home-Schooling (2021), in: SSRN Electronic Journal https://ssrn.com/abstract=3795639 [abgerufen am 14.07.2021]. 
Pschyrembel online: Stichwort Internalisierende Störung (2016), https://www.ps chyrembel.de/Internalisierende\%20St\%C3\%B6rung/P05CP [abgerufen am 24.05.2021].

Pschyrembel online: Stichwort Externalisierende Störung (2016), https://www.ps chyrembel.de/Externalisierende\%20St\%C3\%B6rung/P01SM [abgerufen am 24.05.2021].

Schröder, Martin: Was macht mich zufrieden?, in: Gehirn \& Geist 6 (2021), 13-20.

Rosenfeld, Daniel L./Tomiyama, A. Janet: Can a Pandemic Make People More Socially Conservative? Political Ideology, Gender Roles, and the Case of COVID-19 (2020), https://doi.org/10.31234/osf.io/zg7s4 [abgerufen am 14.07.2021].

Statistik Austria: Familien 1961 bis 2018 nach Familientyp und Kinderzahl (2018), https:/www.statistik.at/web_de/statistiken/menschen_und_gesellschaft/bevoelk erung/volkszaehlungen_registerzaehlungen_abgestimmte_erwerbsstatistik/famil ien/index.html [abgerufen am 24.05.2021].

Stoppacher, Peter/Saurug, Manfed: Armut in der Steiermark. Eine Bestandsaufnahme in unterschiedlichen Bereichen, hrsg. vom Land Steiermark (2018), https://w ww.soziales.steiermark.at/cms/dokumente/12688727_5339/d6cf0b9e/Armutsberi cht\%202018.pdf [abgerufen am 14.07.2021]. 
